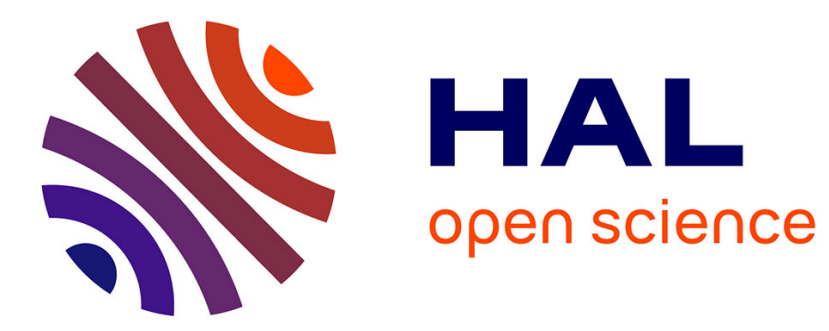

\title{
Sparse EEG source localization in frequency domain
}

Viviana del Rocío Hernández-Castañón, Steven Le Cam, Radu Ranta

\section{To cite this version:}

Viviana del Rocío Hernández-Castañón, Steven Le Cam, Radu Ranta. Sparse EEG source localization in frequency domain. 43rd Annual International Conference of the IEEE Engineering in Medicine and Biology Society, EMBC 2021, Oct 2021, Guadalajara, Mexico. hal-03464026

\section{HAL Id: hal-03464026 \\ https://hal.science/hal-03464026}

Submitted on 2 Dec 2021

HAL is a multi-disciplinary open access archive for the deposit and dissemination of scientific research documents, whether they are published or not. The documents may come from teaching and research institutions in France or abroad, or from public or private research centers.
L'archive ouverte pluridisciplinaire HAL, est destinée au dépôt et à la diffusion de documents scientifiques de niveau recherche, publiés ou non, émanant des établissements d'enseignement et de recherche français ou étrangers, des laboratoires publics ou privés. 


\title{
Sparse EEG source localization in frequency domain
}

\author{
Viviana del Rocío Hernández-Castañón, Steven Le Cam and Radu Ranta
}

\begin{abstract}
This work presents an approach for EEG source localization when strong priors on predominant frequencies in the activities of the source are available. We describe the fundamentals of the used source reconstruction method based on a greedy approach, which can be applied indifferently in the time or frequency domain. The method is evaluated using simulated data reproducing realistic recorded activities in the context of fast periodic visual stimulation. In particular the advantage of reconstructing the source in the frequency domain against time domain is quantified in a realistic setup. Finally, the performances of the method are illustrated on real EEG signals recorded during a fast periodic visual stimulation task.

Index Terms-EEG source localization, frequency domain, sparse inverse problem
\end{abstract}

\section{INTRODUCTION}

One of the most widely used techniques for exploring the electrical brain activity is the electroencephalogram (EEG). The EEG electrodes, placed on the surface of the head, capture potentials generated by current sources situated inside the brain. EEG source estimation and localization implies a wide panel of techniques to reconstruct the source map and their activities [1]. Most importantly, when determining the sources that generate the EEG signal, constraints have to be incorporated in order to constrain and regularize this highly ill-posed inverse problem. In particular, we are interested, for reasons explained below, in approaches enforcing sparsity, for which the main hypothesis is that the recorded signals are generated by few active regions, possibly organized in a network.

While classical source localization methods are developped for time-domain signals, in some situation the relevant information lies in specific frequency bands. Very few approaches were proposed directly in the frequency domain ( [2]-[4]), see also [1] for a brief review. Besides sparsity, we narrow down our interest to this particular situation, i.e., when sources of interest are band-limited or even pure oscillations.

Indeed, our target application is source reconstruction during fast periodic visual stimulation of the brain (FPVS).

FPVS is a privileged technique to study the brain face recognition system, as it is well controlled and provides highly reproducible responses [5] with strong SNR. This kind of protocol consists in a periodic presentation of natural images to the observer at a given base frequency $(6 \mathrm{~Hz}$ for the protocol used in this work), periodically interleaved with face images (at an oddball frequency: $1.2 \mathrm{~Hz}$ in this study,

All Authors are with the Centre de Recherche en Automatique de Nancy (CRAN UMR 7039, University of Lorraine-CNRS, F-54000). radu.ranta@univ-lorraine.fr i.e., every 5 images). Combined with invasive electrophysiological measurements (intracerebral EEG, also known as SEEG), these protocols allowed to identify the main areas recruited for face recognition along the ventral occipitotemporal cortex with a right predominance [5]. These areas synchronize their activities with the visual stimuli, both at the base and the oddball frequencies as illustrated in fig. 3 .

We address in this paper the problem of reconstructing the responsive sources to such protocols from scalp EEG measurements, by taking benefit of the oscillatory nature of the sources of interests and of the fact that they are well localized in specific brain areas. We focus thus on frequency localized and sparse approaches. More precisely, we adopt in this work a method derived from the Matching Pursuit principle used for sparse localization in EEG called Single Best Replacement (SBR) [6] already applied on EEG for time-frequency based localizations [7].

In the present work, the reconstruction performances are analyzed from two angles: by applying the sparse source localization methodology in the time domain after filtering in the frequency bands of interests, as well as directly in the frequency domain after a FFT transform of the recorded channels.

\section{Methodology/Periodic Source localization}

The EEG forward model consists in calculating the electrical potential observed on a given sensor when the configuration of the underlying electrical sources and the biophysical properties and geometry of the propagation environment are known [8]. A general linear forward model is based on a gain matrix, known as lead-field, which encodes the propagation coefficients between a freely oriented source at any position in the head and the measuring electrodes. A simplified model considers fixed orientation sources (i.e. for every possible position only one orientation is considered). Although restrictive, this simplified lead-field can be justified by biological considerations: dipolar current sources represent population of pyramidal neurons, which are not freely oriented but mostly orthogonal to the gray matter surface. Thus, a linear forward model projecting $N$ sources to $M$ electrodes expresses scalp recordings $\mathbf{X}$ as a linear combination of source amplitudes $\mathbf{S}$ through the propagation coefficients stored in the lead-field $M \times N$ matrix $\mathbf{A}$, plus noise $\varepsilon$. Every column of $\mathbf{A}$ corresponds to a specific source position (i.e., contains the propagation coefficients between that specific position and the electrodes).

$$
\mathbf{X}=\mathbf{A S}+\varepsilon
$$


The linear forward model in (1) is generally expressed in the time domain, meaning that $\mathbf{X}$ and $\mathbf{S}$ are, respectively, measurements and source amplitudes at different time instants (matrices $M \times T$ and $S \times T$, with $T$ the number of time samples). But model (1) is valid under any linear transformation of the signals such as the Fourier transform or linear filtering in specific frequency bands. Moreover, the model is valid for any specific time instant, when considered in time, as well as for any specific frequency bin (or band after band-pass filtering), when considered in frequency.

Since the lead-field or gain matrix $A(M \times N, M<N)$ has a number of electrodes $M$ much smaller than the number of possible source locations $N$, the system does not have a unique solution and constraints need to be imposed in order to reduce the solution space. Under sparsity regularization, the estimates of the source amplitudes and of their localization are given by (2):

$$
\hat{\mathbf{S}}, \hat{J}=\underset{\mathbf{S}, J}{\operatorname{argmin}}\left(\left\|\mathbf{X}-\mathbf{A}_{J} \mathbf{S}\right\|_{2}^{2}+\lambda N_{J}\right)
$$

where $J$ contains the indices of the selected columns from the lead-field $\mathbf{A}$, representing source positions, $N_{J}$ represents the number of selected columns (cardinal of $J$ ), $\mathbf{S}$ are the magnitudes of the (selected) sources and $\mathbf{A}_{J}$ is the submatrix containing the $J$ indexed columns from $\mathbf{A}$ (hats indicate estimates of the indices $J$ and source amplitudes $\hat{\mathbf{S}}$; estimating $J$ is equivalent to choosing an estimated submatrix $\hat{\mathbf{A}}_{J}$ ).

In [6], the minimization problem (2) was addressed by a penalized Orthogonal Least Squares (OLS) regression called Single Best Replacement (SBR): the first term decreases with every new added source (index in $\hat{J}$, thus column in $\mathbf{A}_{J}$ and respective magnitude in $\hat{\mathbf{S}}$ ), while the (second) penalization term increases with a fixed amount defined by the user parameter $\lambda$. The algorithm, equivalent to classical OLS for $\lambda=0$, iterates by adding or removing sources until convergence to a more or less sparse solution depending on the user choice of $\lambda$. While the original SBR is applied on vectors (i.e., $\mathbf{X}$ and $\mathbf{S}$ are column vectors), we present here two versions applied on matrices:

- Time domain. In this case, $\mathbf{X}$ is a matrix constructed as follows: (1) band-pass filter the EEG signals (either at $1.2 \mathrm{~Hz}$ or at $6 \mathrm{~Hz}$, see above) using narrow band $(0.2 \mathrm{~Hz})$ 2nd order Butterworth filters; (2) define epochs based on the FPVS frequencies (base or oddball, i.e., 1/1.2 or $1 / 6$ seconds) and average them to reduce the noise. The final $\mathbf{X}$ matrix contains on every row a band-pass averaged signal having the length of one epoch;

- Frequency domain. After Fourier transforming the signals of every electrode, select the complex values of the FFT at every frequency of interest (e.g., $1.2 \mathrm{~Hz}$ and harmonics or $6 \mathrm{~Hz}$ ) to construct the frequency domain $\mathbf{X}$ complex matrix. Note that both positive and negative frequencies need to be considered, as the FFT has hermitian symmetry and both are needed in order to avoid phase indetermination.

Applying the matrix version of the SBR will then yield real time or complex frequency amplitudes $\hat{\mathbf{S}}$ and, most importantly, the set of indices $\hat{J}$ that will allow source localization.

\section{RESULTS}

\section{A. Data simulation}
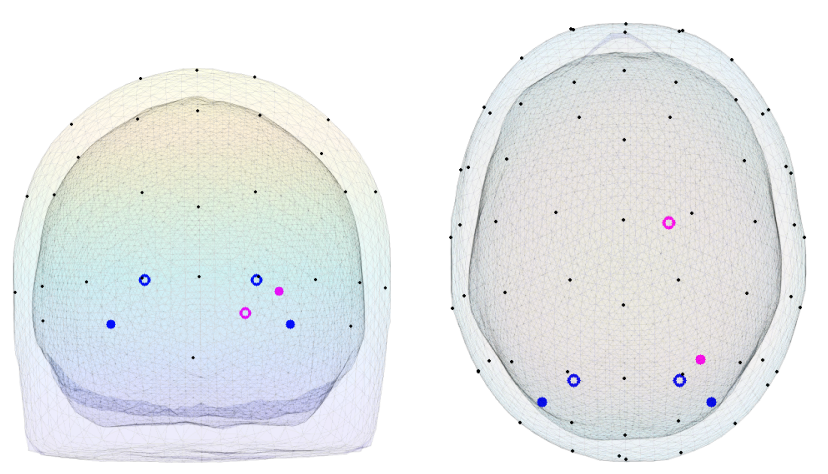

Fig. 1: Simulation setup. In red, oddball sources $(1.2 \mathrm{~Hz})$; in blue, base frequency sources $(6 \mathrm{~Hz})$. The filled circles correspond to $\mathbf{C 1 / C 3}$, empty circles to supplementary sources in C2/C4 (see text). The 64 electrodes are represented as dots.

In order to test the feasibility of the source localization method, EEG scalp potentials were simulated by using a realistic head model with three comparments (Colin 27) extracted from Brainstorm toolbox [9]. The cortical layer mesh (inner shell) has 7292 nodes, the outer skull and the scalp mesh (outer shell) have 1922 nodes. The source space consisted of a regularly spaced grid $(7 \mathrm{~mm})$ constructed inside the inner shell, which yielded $N=6184$ source positions. The scalp electrodes $(M=64)$ were simulated according to the approximate 10-10 system of a Biosemi Cap. The leadfield matrix A was constructed using fixed orientations for all sources, orthogonal to the surface of the head. The EEG scalp potentials were simulated by projecting the sources on the sensors using a boundary element model (BEM) implemented in the Helsinki toolbox [10].

Realistic EEG signals during FPVS contain peaks at oddball and base frequencies, i.e., $1.2 \mathrm{~Hz}$ and $6 \mathrm{~Hz}$ (and harmonics), being thus a mixture of sources at all these frequencies. But for our purposes, as the localization is performed either after band-pass filtering or directly on the peaks of interest after Fourier transform, the actual combination (the respective contributions) of oddball and base frequencies is irrelevant, the important difficulties coming from the interactions between sources having the same frequency. We have simulated different configurations of sources as follows (see fig. 1):

C1 A single sinusoidal source at $f_{o}=1.2 \mathrm{~Hz}$, placed in the right hemisphere, at the approximate location of one of the face responding regions on the posterior ventral visual stream.

C2 Two sources at $f_{o}=1.2 \mathrm{~Hz}$, the one used in $\mathbf{C 1}$ plus a second one, approximately corresponding to a face responding region in the right anterior ventral visual 
stream, about $8 \mathrm{~cm}$ far from the first $1.2 \mathrm{~Hz}$ source and deeper in the brain. This second source was delayed with respect to the first $1.2 \mathrm{~Hz}$ source (phase delay $\pi / 6$ ).

C3 Two sinusoidal sources (same frequency $f_{b}=6 \mathrm{~Hz}$, same phase) were placed symmetrically in left and right hemispheres, approximately in the primary visual areas in the posterior part of the brain. The distance between them was $8 \mathrm{~cm}$.

C4 Four $f_{b}=6 \mathrm{~Hz}$ sources, the two from $\mathbf{C 3}$ and two more, also symmetric between the two hemispheres, but closer one to the other $(5 \mathrm{~cm})$ and each one relatively close to the $6 \mathrm{~Hz}$ sources used in $\mathbf{C 3}(3 \mathrm{~cm})$. They were also synchronized between them (same phase) and slightly delayed with respect to the other $6 \mathrm{~Hz}$ sources (phase delay $\pi / 10)$.

The amplitudes of all sources were identical (but their contribution to the scalp EEG varied of course due to the lead-field coefficients).

We have simulated increasingly complex signals by combining projections of the source configurations described above (4 types of EEG, from one 1.2 and two $6 \mathrm{~Hz}$ sources to two 1.2 and four $6 \mathrm{~Hz}$ sources). For each combination, white Gaussian noise was added to the simulated scalp potentials. Seven noise power levels have been used, computed so as to reach SNRs in the set $\{20 \mathrm{~dB}, 10 \mathrm{~dB}, 3 \mathrm{~dB}, 0 \mathrm{~dB},-3 \mathrm{~dB}$, $10 \mathrm{~dB},-20 \mathrm{~dB}\}$ with respect to the projection of the first $1.2 \mathrm{~Hz}$ source (C1). One hundred simulations per type of EEG and noise level were performed using the set up described previously.

\section{B. Performance criteria on simulated EEG data}

To compare the performance of the localization procedures, two criteria were used: the Goodness of Fit (GOF, computed with respect of the no-noise simulation) and a weighted distance of localization error (wDLE). The GOF is defined as:

$$
G O F=1-\frac{\left\|\mathbf{A}_{J} \mathbf{S}-\hat{\mathbf{A}}_{J} \hat{\mathbf{S}}\right\|^{2}}{\left\|\mathbf{A}_{J} \mathbf{S}\right\|^{2}}
$$

where $\mathbf{A}_{J} \mathbf{S}$ are the simulated electrical potentials without noise (see 1) and $\hat{\mathbf{A}}_{J} \hat{\mathbf{S}}$ are the estimated potentials calculated as the projection of the reconstructed sources on the EEG electrodes. The higher the GOF, the closest is the estimation to the originally simulated data.

We also propose an original weighted distance criterion derived from the Distance of Localization Error (DLE) [11], where the source distances are weighted by their contributions to the reconstructed potentials:

$$
w D L E=\frac{\sum_{j \in J} \alpha_{j} \min _{i \in I}\left\|r_{j}-r_{i}\right\|}{2 \sum_{j \in J} \alpha_{j}}+\frac{\sum_{i \in I} \alpha_{i} \min _{j \in J}\left\|r_{i}-r_{j}\right\|}{2 \sum_{i \in I} \alpha_{i}}
$$

$J$ is the set of the $N_{J}$ reconstructed sources at positions $\left\{r_{j}\right\}_{j \in\left[1 \ldots N_{j}\right]}$, and $I$ is the set of the $N_{I}$ simulated sources placed at positions $\left\{r_{i}\right\}_{i \in\left[1 . . N_{i}\right]}$. The coefficient $\alpha_{j}$ is computed as the norm of the projection of the $j^{\text {th }}$ estimated source on the electrodes divided by the norm of the reconstructed scalp map. Similarly, $\alpha_{i}$ is the weight given by the ratio of the norm of the potential due to the $i^{\text {th }}$ simulated source with respect to the norm of the potential of the simulated data. The first term of equation (4) penalizes the presence of false positive sources, while the second term penalizes true sources that are not recovered by the method.

\section{Simulation results}

The figure 2 sums up the performance in term of true positives (TP)/false positives (FP) and GOFs, and table I provides averages values of wDLE for each configuration.

TABLE I: Mean \pm std wDLE values of the source localization in frequency and time domain for the four source configurations (in $\mathrm{mm}$; 100 simulations per SNR)

\begin{tabular}{|c|c|c|c|c|}
\cline { 2 - 5 } \multicolumn{1}{c|}{} & \multicolumn{2}{c|}{ C1 } & \multicolumn{2}{c|}{ C2 } \\
\hline $\begin{array}{c}\text { SNR } \\
(\mathbf{d B})\end{array}$ & Frequency & Time & Frequency & Time \\
\hline 20 & $0 \pm 0$ & $0 \pm 0$ & $0.02 \pm 0.01$ & $0.08 \pm 0.07$ \\
\hline 10 & $0 \pm 0$ & $0 \pm 0$ & $0.13 \pm 0.40$ & $1.36 \pm 2.57$ \\
\hline 3 & $0 \pm 0$ & $0.75 \pm 1.52$ & $1.19 \pm 2.22$ & $4.25 \pm 4.09$ \\
\hline 0 & $0 \pm 0$ & $3.22 \pm 3.90$ & $1.81 \pm 3.03$ & $7.13 \pm 4.16$ \\
\hline-3 & $0 \pm 0$ & $10.07 \pm 8.12$ & $3.02 \pm 3.76$ & $11.11 \pm 4.67$ \\
\hline-10 & $1.21 \pm 2.24$ & $35.75 \pm 11.71$ & $7.07 \pm 4.08$ & $25.07 \pm 7.68$ \\
\hline-20 & $34.93 \pm 11.69$ & $47.57 \pm 8.29$ & $24.11 \pm 6.90$ & $38.54 \pm 4.75$ \\
\hline \multicolumn{3}{|c|}{ C3 } & \multicolumn{2}{c|}{ C4 } \\
\hline $\begin{array}{c}\text { SNR } \\
(\mathbf{d B})\end{array}$ & Frequency & Time & Frequency & Time \\
\hline 20 & $0.04 \pm 0.02$ & $0.05 \pm 0.02$ & $18.06 \pm 0.01$ & $18.06 \pm 0.01$ \\
\hline 10 & $0.14 \pm 0.05$ & $0.16 \pm 0.07$ & $18.06 \pm 0.02$ & $18.06 \pm 0.04$ \\
\hline 3 & $0.30 \pm 0.12$ & $0.35 \pm 0.15$ & $18.03 \pm 0.24$ & $17.92 \pm 0.61$ \\
\hline 0 & $0.42 \pm 0.16$ & $0.48 \pm 0.21$ & $17.96 \pm 0.67$ & $17.87 \pm 1.02$ \\
\hline-3 & $0.58 \pm 0.22$ & $0.65 \pm 0.28$ & $17.98 \pm 0.90$ & $17.96 \pm 1.04$ \\
\hline-10 & $2.62 \pm 3.39$ & $4.84 \pm 5.72$ & $18.17 \pm 1.31$ & $18.66 \pm 1.44$ \\
\hline-20 & $25.79 \pm 10.82$ & $30.38 \pm 12.19$ & $19.82 \pm 2.00$ & $21.28 \pm 5.24$ \\
\hline
\end{tabular}

When a single superficial source is simulated for a given frequency (C1), the method is robust and tends to fail for strong noise only: the localization in frequency domain is accurate up to $-10 \mathrm{~dB}$ SNR, with the low wDLE and high GOFs (near 1 ), very few to no FP, while in the time domain the performance becomes weaker from the $-3 \mathrm{~dB}$ case (about $2 \mathrm{FP}$ and wDLE over $1 \mathrm{~cm}$ on average for this noise level). When two $f_{o}$ simulated sources are introduced (C2), the method clearly struggles in recovering the deeper (second) source, the frequency approach being accurate up to $-3 d B$, while in the time domain the performances are mild from $3 \mathrm{~dB}$ SNR, where the number of FP is near 2 with associated GOF values over $30 \%$ on average. It is worth noticing that the localization based on FFT produces globally much less FP in these two configurations of $f_{o}$ sources compared to the time domain.

Considering the reconstruction of the $f_{b}=6 \mathrm{~Hz}$ sources, the inversion in frequency and time domain do not differ significantly. When two $f_{b}$ sources are simulated (C3), the reconstruction is accurate over all SNRs (putting the $-20 d B$ case aside). It can be seen that about two FP are consistently found, but with very low amplitude and thus with unsignificant impact on the reconstructed data (low 

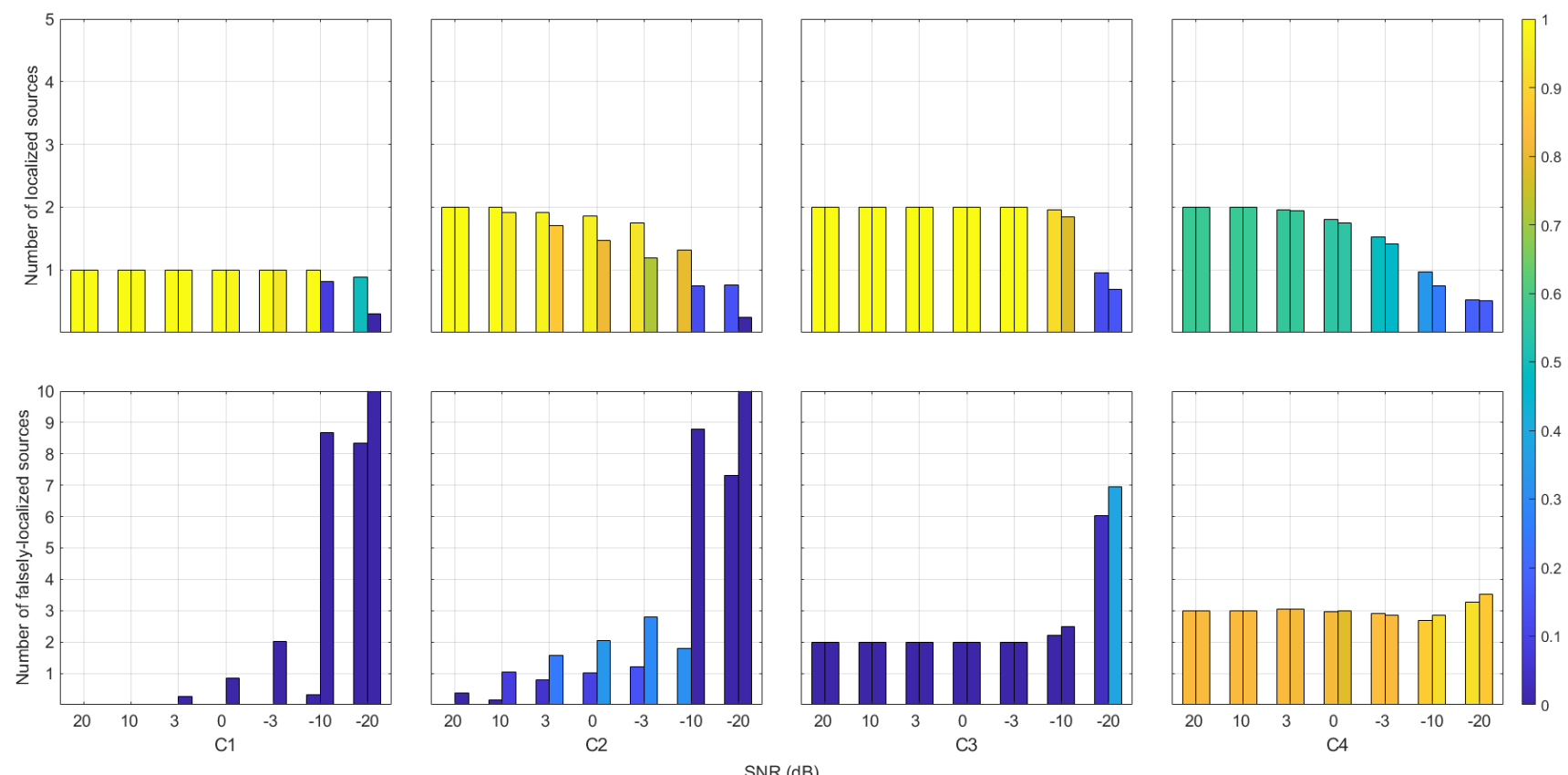

Fig. 2: Source localization performances. Top row: number of true positives (correctly localized sources, within $1 \mathrm{~cm}$ from the simulated source). Bottom row: number of false positives (falsely-localized sources). The results are displayed for the four source configurations, for different levels of SNR. For every SNR, the first bar shows the performance in frequency domain and the second bar in time domain. The color represents the accuracy of reconstruction (GOF), see colorbar.

FP GOFs and low wDLE). However when four $f_{b}$ sources are simulated (C4), the method is failing even in the most favorable $20 d B$ SNR case. Let recall that the first and second groups of two sources are synchronized two by two and slightly delayed between the two groups, and that they are all placed in the occipital areas. This becomes infeasible for the method to distinguish between them as they are relatively close in a region where the EEG sensors are only partly covering the projection of the dipoles. A closer look at the algorithm first iterations reveals that the algorithm is misled from the beginning by choosing false sources explaining a high portion of the data (see the high GOFs for the FP on fig.2.C4, bottom), and the SBR strategy is not able to discard them afterwards.

To sum up, when the method is able to recover the sources, a clear advantage is given to the inversion in the frequency domain (C1 and C2 configurations), as the method focused on the frequency value fully supporting the information and as the temporal filtered version of the data still collects some noise residuals. Such strategy is helpful to recover profound sources, an important aspect when the reconstruction is carried out from the EEG measurements. In some case of source configurations where the source map is not identifiable by the method (e.g., close and synchronised activities), the inversion in the frequency domain is not helpful in disentangling the contributions of the sources.

\section{Real signals}

An example of the frequency based localization method is given here, on a 64-channels real EEG recording (BioSemi
Cap) at a sampling rate of $256 \mathrm{~Hz}$. Two approximately 1 minute long sessions were recorded using the protocol described in the introduction [5]. After average re-referencing, two sessions averaging and Fourier Transform, one can obtain spectra like the one presented fig. 3, with peaks at the oddball frequency of $1.2 \mathrm{~Hz}$ and harmonics $(2.4 \mathrm{~Hz}$, $3.6 \mathrm{~Hz}$, etc.). This particular spectrum was recorded on the PO8 electrode (see also the power scalpmap at $1.2 \mathrm{~Hz}$, same figure). One can also notice an important peak in the spectrum at the base frequency $(6 \mathrm{~Hz})$.

The frequency localization method was performed after selecting in the $\mathbf{X}$ matrix (1) the complex values of the FFT corresponding to the peaks at $1.2,2.4 \mathrm{~Hz}$ and $3.6 \mathrm{~Hz}$ and their negative counterparts. We used the same head model as for the simulated signals (lead-field matrix A). The result, after tuning $\lambda$ to the norm of $\mathbf{X}$ divided by 20, are shown fig 3 . Three sources were reconstructed $(\mathrm{GOF}=0.88$ with respect to the measured data $\mathbf{X}$ ) in the posterior brain region and both hemispheres (results to be compared for example with the fMRI images from [12].fig.2). Of course, these results should be interpreted with caution. For example, the leadfield matrix that we used is designed for the simulation, but not optimized for the subject on which the EEG recordings were performed (nor actually optimized for sources in the gray matter with more carefully selected orientations).

\section{CONCLUSiON}

In this paper, we have proposed a sparse source estimation method in the context of oscillatory source activities, in response to periodic stimulation as observed e.g., in FPVS. 

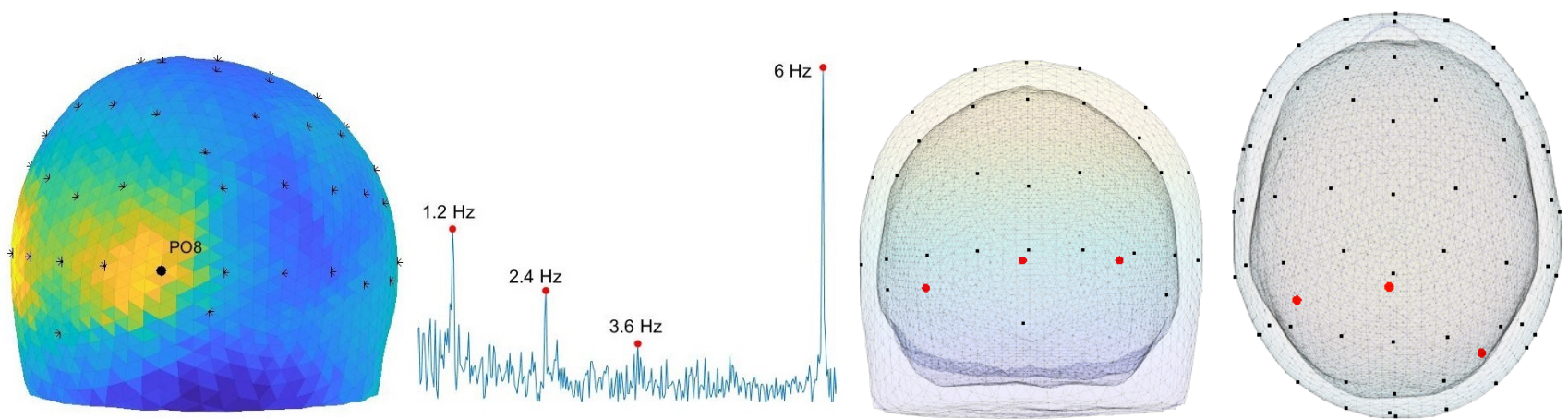

Fig. 3: Frequency source localization in real signals. Three sources were reconstructed (red dots) from a data vector with the peaks at the frequencies 1.2, 2.4 and $3.6 \mathrm{~Hz}$. The scalpmap at $1.2 \mathrm{~Hz}$ is given at the left, centered on the PO8 electrode, for which the spectrum is displayed nearby.

The method can be applied on either an epoch-averaged band-pass filtered version of the signal centered on the frequency of interest or directly on the FFT of the signal, by considering the complex values associated with the peaks at these frequencies.

We have demonstrated on simulation the higher robustness of this former version, as the information of interest is maximally concentrated in these peaks. In particular, this approach is likely to provide more reliable reconstruction of deep brain sources from the EEG surface measurements. However, our simulation results also illustrate that the reconstruction of synchronized sources concentrated in a limited area of the brain is a highly challenging task for the proposed iterative method, whatever the chosen reconstruction domain. The method fails and converges to an equivalent solution with high GOF but wrong source estimates, which brings us back to the initial question of the regularization of this severely ill-posed inversion problem. In order to solve such indeterminacy, it appears to be necessary to introduce into the method additional neurophysiological or anatomical constraints on the position of the sources.

We finally illustrate our methodology on an example of real data recorded during an FPVS protocol. The results, which need to be further analysed and evaluated using a brain atlas and a wider panel of subjects, is nevertheless coherent with the present knowledge on brain face responsive areas and is a motivation to pursue our research in this direction.

\section{ACKNOWLEDGMENT}

The authors would like to thank Angélique Volfart for her cooperation in providing the real EEG data and for her comments. This work was partly supported by funding from the National Council of Sciences and Technology of Mexico (CONACyT).

\section{REFERENCES}

[1] C. Michel et al., "EEG source imaging," Clinical neurophysiology, vol. 115, no. 10, pp. 2195-2222, 2004.

[2] P. A. Muñoz-Gutiérrez et al., "Localization of active brain sources from EEG signals using empirical mode decomposition: A comparative study," Frontiers in integrative neuroscience, vol. 12, p. 55, 2018.

[3] X. Zheng and B. Wan, "Study on EEG source localization in frequency domain," in 27th IEEE EMBC Annual Conference, 2005, pp. 5958-5961.

[4] B. Lütkenhöner, "Frequency-domain localization of intracerebral dipolar sources," Electroencephalography and clinical Neurophysiology, vol. 82, no. 2, pp. 112118, 1992.

[5] J. Jonas et al., "A face-selective ventral occipitotemporal map of the human brain with intracerebral potentials," Proceedings of the National Academy of Sciences, vol. 113, no. 28, pp. E4088-E4097, 2016.

[6] C. Soussen et al., "From Bernoulli-Gaussian deconvolution to sparse signal restoration," IEEE Transactions on Signal Processing, vol. 59, no. 10, pp. 4572-4584, 2011.

[7] G. Korats et al., "A space-time-frequency dictionary for sparse cortical source localization," IEEE Transactions on Biomedical Engineering, vol. 63, no. 9, pp. 19661973, 2016.

[8] H. Hallez et al., "Review on solving the forward problem in EEG source analysis," J Neuroeng Rehabil., pp. 4-46, 2007.

[9] S. Baillet et al., "BrainStorm: a Matlab Toolbox for the Processing of MEG and EEG Signals," Neurolmage, vol. 9, pp. S246-S246, 1999.

[10] M. Stenroos, V. Mäntynen, and J. Nenonen, “A Matlab library for solving quasi-static volume conduction problems using the boundary element method," Computer methods and programs in biomedicine, vol. 88, no. 3, pp. 256-263, 2007.

[11] H. Becker et al., "A performance study of various brain source imaging approaches," in IEEE International Conference on Acoustics, Speech and Signal Processing (ICASSP). IEEE, 2014, pp. 5869-5873.

[12] T. C. Kietzmann et al., "The occipital face area is causally involved in facial viewpoint perception," Journal of Neuroscience, vol. 35, no. 50, pp. 16398-16403, 2015. 